\title{
Technology of a regional Guthrie test service
}

\author{
R. L. NEWMAN AND D. J. T. STARR \\ From Queen Mary's Hospital for Children, Carshalton, Surrey
}

SYNOPSIS Investigations were undertaken to discover the effect and importance of technical factors in the Guthrie test and to determine the most satisfactory materials available.

These findings were used in setting up the screening service for phenylketonuria for the South West Metropolitan Region.

The Medical Research Council Working Party on phenylketonuria (1968) recommended that the Guthrie test should be adopted on a national scale to replace the Phenistix test as a screening procedure for phenylketonuria.

Recent evidence (Hudson, Mordaunt, and Leahy, 1970) has confirmed the value of early dietetic treatment and the need for early diagnosis, and Guthrie test services are being developed in most parts of the United Kingdom. This test has been in routine use for three years in this laboratory which deals with 50,000 specimens yearly for the South West Metropolitan Region.

The experience gained in the early pilot studies with the Guthrie test and subsequently in developing a regional service is presented here from a laboratory point of view. Investigations were undertaken to discover the effect and importance of factors which influenced the test and to determine the best materials to use. Administrative problems are outside the scope of this paper and are discussed in a separate publication.

\section{Collection Cards}

Blood samples are collected from babies by heel prick and absorption of the blood onto specially prepared blotting paper. Several printed commercial collection papers are available and range from simple cut papers to those made into pads with interleaved carbon paper for recording details. Most of those examined were expensive and unnecessarily complicated. The sequential numbering available on some seemed of little value when cards were to be returned randomly from numerous centres. Cards with carbon paper proved unsatisfactory as handling made the samples very dirty.

Received for publication 5 November 1970.
Simplicity appeared of paramount importance and we make a card ( 3 in. $\times 2$ in.) of suitable paper $c$ with three printed circles $\frac{3}{8}$ in. diameter to indicate $\frac{\mathbb{D}}{O}$ the quantity of blood required and with spaces for $\stackrel{\mathbb{D}}{\mathcal{S}}$ the surname, the date of collection, and a code number. The address can be written on the back. $\stackrel{\Phi}{~}$ Polythene envelopes are necessary to protect the $\vec{\theta}$

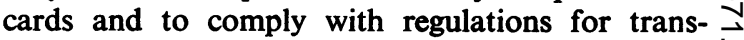
mission of pathological specimens by post.

\section{Paper}

The quantity of blood contained in a disc of paper of given size varies with the type of paper and standard discs must be made of the same paper as that used for collecting samples. The paper must be highly absorbent to allow for rapid and uniform spotting of blood, and must be thick enough to soak up a useful quantity. Guthrie and Susi (1963) gave as a likely source of error in certain published studies (Scheel and Berry, 1962) the choice of Whatman 3MM paper. This has a hard surface and absorbs blood only slowly. Guthrie used Schleicher and Schuell 903 but, because of the cost and difficulties of supply of this American product, an alternative readily available in this country was sought.

Many manufacturers and suppliers of paper provided information and samples. Seven papers $\Omega$ were examined and the mean weight of a 1 in. disc $N$ from each was recorded. Standard volumes of blood were applied slowly to each paper and the mean diameters of the spots were measured. The weights of dried blood contained in 1 in. discs from these spots were determined. The results are shown in Table I.

Blood with a phenylalanine concentration of approximately $8 \mathrm{mg} / 100 \mathrm{ml}$ was spotted onto each paper. Discs from the dried spots were used for 564 


\begin{tabular}{|c|c|c|c|}
\hline Paper & $\begin{array}{l}\text { Weight of } \\
\frac{1}{\text { tin. Paper }} \\
\text { Disc (mg) }\end{array}$ & $\begin{array}{l}\text { Weight of } \\
\text { Dried Blood } \\
\text { per Disc } \\
\text { (mg) }\end{array}$ & $\begin{array}{l}\text { Diameter of } \\
\text { Blood Spots } \\
(\text { mm }) \text { with } \\
\text { Standard } \\
\text { Blood Volumes } \\
(\text { approx } \\
50 \mathrm{cmm})\end{array}$ \\
\hline $\begin{array}{l}\text { Whatman ET } 31 \\
\text { Mast L aboratories } \\
\text { (loose sheets) }\end{array}$ & $\begin{array}{l}6 \cdot 2 \\
9 \cdot 6\end{array}$ & $\begin{array}{l}2 \cdot 7 \\
4 \cdot 0\end{array}$ & $\begin{array}{l}12 \cdot 5 \\
11 \cdot 0\end{array}$ \\
\hline $\begin{array}{l}\text { Mast Laboratories } \\
\text { (carbon paper pads) }\end{array}$ & 5.9 & $2 \cdot 7$ & $12 \cdot 5$ \\
\hline Bacto-Difco & 4.9 & $1 \cdot 3$ & $13 \cdot 0$ \\
\hline $\begin{array}{l}\text { E. Merck } \\
\text { Department of Health card }\end{array}$ & $6 \cdot 5$ & $2 \cdot 5$ & $12 \cdot 5$ \\
\hline $\begin{array}{l}\text { HMR 101/6 } \\
\text { (Schleicher \& Schuell 903) }\end{array}$ & $5 \cdot 7$ & $2 \cdot 6$ & $13 \cdot 0$ \\
\hline Whatman $3 / M M$ & 5.9 & $2 \cdot 3$ & $12 \cdot 5$ \\
\hline
\end{tabular}

Table I Comparison of papers available for Guthrie test ${ }^{1}$

${ }^{1}$ Mean values are given

Guthrie tests and the growth zone diameters were measured.

As anticipated, from a comparison of the amounts of dried blood contained in discs from each paper, the growth zone diameters showed a variation between papers (minimum $17 \mathrm{~mm}$, maximum $21 \mathrm{~mm}$ ). This would be equivalent to results of $6 \mathrm{mg} / 100 \mathrm{ml}$ and $12 \mathrm{mg} / 100 \mathrm{ml}$ respectively. The maximum variation for any individual paper, however, was $2 \mathrm{~mm}$ (Whatman 3MM). All the papers absorbed blood rapidly except Whatman 3MM. The results for Whatman ET 31 all fell between $18 \mathrm{~mm}$ and $19 \mathrm{~mm}$. In these experiments and in lengthy trials Whatman ET 31, a thick, very absorbent chromatography paper, has proved satisfactory. It is now in routine use in this laboratory.

The cost of printing circles and words on the collecting papers was found to be high even in large quantities, and satisfactory collecting cards were made by cutting the paper and printing with a rubber stamp using a suitable jig. Many inks were found to have a bacteriostatic effect and some diffuse when autoclaved. Duplicating ink, HMSO 42-47, proved satisfactory.

Cards with high quality printing can be made very cheaply by the use of a simple Adana hand-operated printing machine. The 'eight five' size permits four cards to be obtained from a single block and this method is now used. In this way, adequate reserves of cards, for a service using 1,000 per week, can be produced if printing is carried out on occasional days when the number of test samples received is low.

\section{Department of Health Collection Cards HMR 101/6}

Since September 1969 the Department of Health has issued a printed collecting card made from
Schleicher \& Schuell 903 paper, which was found in the above experiments to have similar properties to Whatman ET 31. The card measures $5 \frac{3}{4}$ in. $\times 4 \frac{1}{4}$ in., has four printed circles $\frac{3}{8}$ in. in diameter for the blood spots, and spaces for a number of details. Supplies of the unprinted paper are also available for those laboratories which prefer an alternative size and format for their test card.

\section{Storage of Samples}

Blood discs were found to be stable by Hill, Summer, Pender, and Roszel (1965) when stored for 27 days at room temperature, and by Searle, Mijuskovic, Widelock, and Davidow (1967) when stored in the dark at $5^{\circ} \mathrm{C}$ in a dessicator over silica gel for at least three months.

Dried standard blood samples on paper stored for many months (cold, dark room in a desiccator over silica gel) gave consistent results when compared with freshly prepared standards. Test samples of blood on paper remain stable for several weeks at room temperature and no deterioration occurs in the relatively short period taken to collect samples, despatch by post, and test at the laboratory.

\section{Preparation of Samples}

If blood discs are placed onto Demain's medium without preliminary treatment, such as autoclaving, diffusion of blood pigment occurs which makes the reading of some results very difficult. Some workers, by manipulation of the amount of inhibitor, avoid autoclaving, but we found the improved ease of reading after autoclaving compensated for the slight extra work. In addition autoclaving destroys certain substances (eg, antibiotics, see later) which can produce varying degrees of inhibition within the growth zone or even complete suppression of growth. This factor alone weighs very heavily in favour of autoclaving specimens and its importance cannot be overemphasized if false negatives are to be avoided.

The use of metal trays and wire racks to hold the collection cards led to problems of condensation during autoclaving. A specially designed nest of wooden trays made from resin-bonded marine grade plywood (Fig. 1) is now routinely used and has survived months of autoclaving with little damage. Each of the four trays in the frame holds 80 test cards plus standards, sufficient for one assay plate, and each sample stands distinct from its neighbour. The cover of the assembly protects the cards from condensation drips, but it is open at the sides to allow free circulation of the air and steam mixture.

Previous accounts refer to a dry steam source 

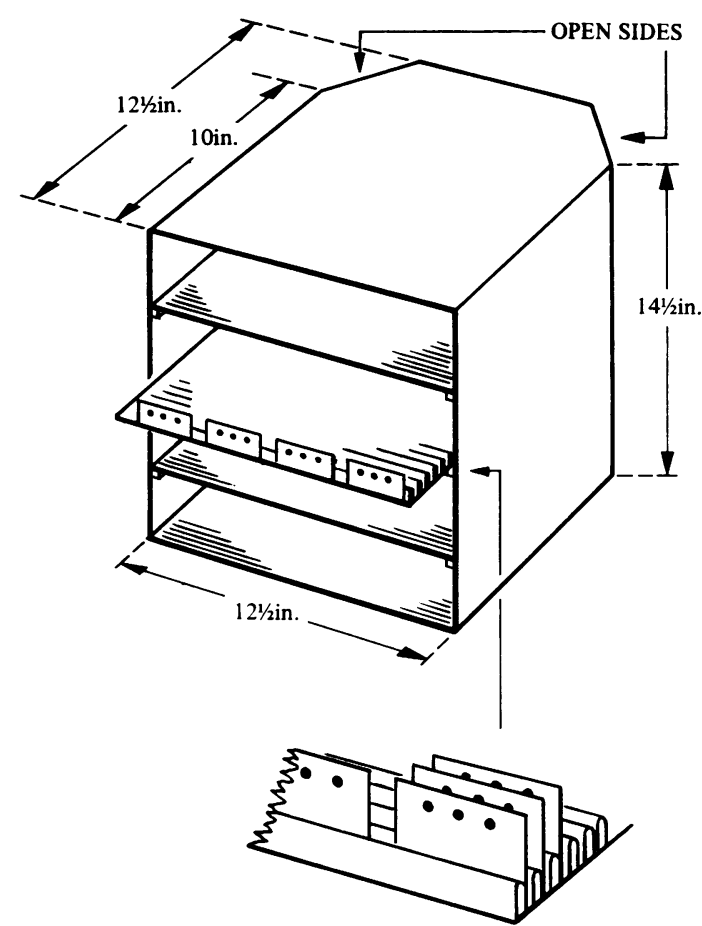

Fig. 1 Diagram of apparatus for holding cards during autoclaving

(15 lb pressure required for three minutes only). Difficulties were encountered in early attempts to autoclave specimens at this pressure for such a short time as most laboratory autoclaves take a long time to reach $15 \mathrm{lb}$ pressure and cards were very wet after such treatment. Exposure to steam and heat for long periods results in loss of phenylalanine. The best results were achieved by the following procedure using a small portable laboratory autoclave: (1) bring water in autoclave to boil; (2) load and seal immediately, when pressure of this air and steam mixture will quickly rise to $5 \mathrm{psi}$; (3) switch off source of heat when 5 psi has been reached; (4) keep exhaust valve closed while autoclave is cooling; (5) remove contents immediately pressure has returned to zero. This treatment is effective in preventing diffusion of haemoglobin while not affecting the phenylalanine level.

\section{Alternatives to Autoclaving for Treatment of Samples}

Efron, Young, Moser, and MacCready (1964) tried protein precipitants such as picric acid, perchloric acid, and trichloroacetic acid before chromatography to prevent interference by blood pigments. Glacial acetic acid has also been used before Guthrie testing
(Lund, personal communication). This method 을 was run in parallel with autoclaving for routine specimens. Cards were hung on glass rods over glacial $\stackrel{\rho}{+}$ acetic acid in a sealed tank for varying lengths of time. They were then removed, and left on the bench for differing times to remove the glacial acetic acid fumes. One hour in the acid fumes was found to be $\triangle$ long enough to prevent diffusion of pigment from $\%$ the test samples of blood without appreciably $\vec{\circ}$ altering the test result. Less than one hour was adequate for the acid fumes to disperse. Table II $\vec{\omega}$ shows the effect of time of exposure and airing of discs.

\begin{tabular}{llll}
\hline $\begin{array}{l}\text { Time of Exposure } \\
\text { of Disc to Glacial }\end{array}$ & Time in Air (min) & $\begin{array}{l}\text { Diffusion of } \\
\text { Pigment }\end{array}$ & $\begin{array}{l}\text { Diameter of } \\
\text { Growth Zone } \\
(\mathrm{mm})\end{array}$ \\
\hline 5 & & + & 18 \\
15 & 120 & \pm & 18 \\
30 & 120 & 0 & 18 \\
45 & 120 & 0 & 18 \\
60 & 120 & 0 & 18 \\
60 & 120 & 0 & 18 \\
& 0 & & (Less dense \\
60 & & 0 & growth) \\
60 & 30 & 0 & 18 \\
60 & 60 & 0 & 18 \\
\hline
\end{tabular}

Table II Effect of glacial acetic acid on blood samples for Guthrie test

In a further series of five experiments 150 negative and 74 elevated samples were tested in parallel by autoclaving and by acetic acid treatment. There was full agreement in the results by both methods.

Disadvantages of the glacial acetic acid method were: (1) the presence of traces of acid could be smelt and felt when handling the cards to punch discs, and this might be unacceptable over a long period. Handling after treatment with glacial acetic acid could be overcome by punching the discs and then treating only the punched disc with the acid fumes, but this method might lead more easily to confusion of specimens. (2) After acid treatment the discs were rather more stiff than after autoclaving and occasionally with a poorly collected blood spot (eg, one with creases) complete contact could only be achieved by pressing the disc very firmly onto the surface of the medium. (3) Occasionally discs showed a zone of inhibition within the zone of growth using the acid method. Subsequent autoclaving of such blood samples resulted in a normal growth zone on retesting. This phenomenon is due to antibacterial substances in the blood and is discussed later under 'antibiotics'.

It was concluded that the autoclaving method was preferable, but the acid method could be useful as an alternative with reservations. 


\section{Dry Heat}

Further experiments were carried out to determine the effect of dry heat in a hot air oven at a temperature of $100^{\circ} \mathrm{C}$ for periods of up to six hours. The dry heat had virtually no effect on phenylalanine level nor did it prevent diffusion of pigment. Prolonged autoclaving, however, had a noticeable effect on the test result. As blood with a phenylalanine level of $10 \mathrm{mg} / 100 \mathrm{ml}$ was used in these experiments, the growth zone could readily be measured despite the diffusion of pigment.

\section{Punching and Transfer of Dises}

Quarter inch discs are punched using perforating pliers and are placed in numerical order in Perspex haemagglutination trays ready for subsequent transfer with forceps to the assay plate. When the test discs have been punched from the collecting cards they are no longer identifiable except by their position in the tray and on the plate. Great care must be taken to avoid misplacement.

Guthrie and Susi (1963) placed the discs onto numbered squares on a sheet of paper for later transfer to the assay plate. They pointed out that the use of a plastic sheet with depressions prevented displacement by air currents. Wainer and Metzger (1967) have described a device for rapid plating of discs, which are held by a small static charge on Lucite, final transfer being achieved by inversion of the assay plate onto the apparatus. Warnings are given regarding draughts and mechanical jarring.

Kennedy (1969) suggested an improved device. A glass plate was substituted for the Lucite sheet and discs were positioned and protected by a plastic sheet with drilled holes. This is a very practical device which speeds the transfer of discs.

\section{Media}

All the commercial preparations obtainable were considered for cost, presentation, availability, and laboratory performance. Samples of medium were examined from Bacto-Difco Ltd, Baltimore Biological Laboratories, Mast Laboratories Ltd, E. Merck A.G., and Oxoid Ltd.

The preparations were available as dehydrated powder, as prepared agar which required re-melting, or as a double-strength agar which needed mixing after melting with a solution of other ingredients. No advantage was noted in this latter method of preparation; and, in addition, purchase of prepared agar in bottles restricted the choice of volume for the assay plate.

All samples gave readable results. The colour of the reconstituted media varied from virtually colourless to a deep orange brown and apparently depended to a great extent on heating in manufacture.

Media were available with or without inhibitor. In some batches tested the amount of inhibitor appeared to be barely adequate and it was necessary to add inhibitor to avoid background haze. This is more fully discussed later but, because of the advantages of carefully controlling the concentration of inhibitor, we preferred medium supplied without inhibitor.

The preparations available as dehydrated powders varied from one which required soaking in cold distilled water and heating for a few minutes in a boiling water bath to one which had to be dissolved in water at $60^{\circ} \mathrm{C}$ followed by autoclaving at $15 \mathrm{lb}$ pressure for 15 minutes.

It was decided that a medium, clear and colourless, prepared from dehydrated powder without inhibitor and requiring the minimum of heating, would be most suitable.

Most of the products are manufactured outside the UK and difficulties were encountered in the supply of some media from the USA. As continuity of supply is essential, a British manufacturer (Oxoid Ltd) was approached and prepared experimental batches of medium to the required specification. This medium can be conveniently prepared on a hotplate. It was found to be very satisfactory and is now available commercially. The method of preparation is given later.

\section{Spores}

The method of preparation of the spore suspension of B. subtilis ATCC 6051 given by Guthrie and Susi (1963) was used with the following modifications. Smith and Gordon (1957) observed improved sporulation of $B$. subtilis when soil extract was added to refined agar. Five $\mathrm{g}$ of garden soil was, therefore, added to $500 \mathrm{ml}$ of tap water, well shaken and allowed to stand overnight. The decanted fluid was used instead of distilled water to prepare 'LabLemco' agar. The medium was sterilized by autoclaving for 15 minutes at $15 \mathrm{lb}$ pressure and plates were poured. After setting, the surface was inoculated heavily with an 18-hour broth culture of $B$. subtilis and incubated overnight. The resulting culture contained a very high proportion of spores and this method has proved satisfactory over a long period.

The spores are harvested and washed as described by Guthrie and Susi (1963) and stored at room temperature in distilled water. Working suspensions are prepared by dilution with distilled water and standardized by trial and error. The dilute suspension is stored at $4^{\circ} \mathrm{C}$ and remains stable for at least several months. 
In the preparation of the medium, the spore suspension is added when the medium has cooled. It was found that the spore suspension could be added at temperatures considerably higher than the $45-50^{\circ} \mathrm{C}$ usually used without detriment to the spores. Ten minutes' boiling during preparation had no visible effect on growth. The temperature now used routinely is $60^{\circ} \mathrm{C}$, which is sufficiently low to avoid excessive condensation on assay plate lids but gives generous working time to pour plates. This higher temperature may have some advantages in reducing contamination of plates and allows more time for better mixing of spores.

The medium may be used soon after preparation or may be kept at $4^{\circ} \mathrm{C}$ for a few days. There is a gradual deterioration in the quality of prepared plates with storage, and contamination with moulds may show after a few days.

\section{Inhibitor}

In an inhibition assay many factors interact and contribute to the final result. The concentration of inhibitor in the medium is critical and affects results more than any other single factor. Increasing the amount of inhibitor results in smaller growth zones and will reduce background haze, but also delays the consolidation of the growth around the disc. It is desirable to adjust the amount of inhibitor to confine the zone of growth from normal blood specimens to diameters which can be easily measured since larger zones tend to have less discrete edges, are difficult to measure, and the difference in zone sizes becomes less. The concentration of inhibitor should be fixed to show some growth from blood specimens with a very low phenylalanine content.

Other workers have varied the amount of inhibitor to give growth/no growth results at levels of 2 or $4 \mathrm{mg} / 100 \mathrm{ml}$ phenylalanine. Experience has shown that a small zone of growth round all discs makes reading of results no more difficult and gives an indication that the test is working satisfactorily Furthermore, it is impossible to detect inhibition of growth by antibacterial substances if the growth/no? growth method is used. We have found a final con-C centration in the medium of $4.0 \times 10^{-5} \mathrm{M}$ satis factory.

In neonatal screening maximum differentiation is required for phenylalanine levels of approximatelyen 4 to $6 \mathrm{mg} / 100 \mathrm{ml}$. With the sensitivity of the tes 5 adjusted to this end, no difficulty is experienced in detecting much higher phenylalanine levels, althoughw the result at high levels is less accurate quantitatively

Another important factor affecting zone diametero is the depth of the medium. This factor also in $-\infty$ fluences the length of incubation time for optimumos readability of results. Variations in depth from oneor part of a plate to another may easily affect results. + We have found $200 \mathrm{ml}$ of medium in a $10 \mathrm{in.} \times 10 \mathrm{in.}$. glass and metal assay plate (Mast Laboratories) to $\vec{c}$ be most suitable. This gives a depth of medium off $3 \mathrm{~mm}$ and requires 18 hours' incubation. When $\frac{\overrightarrow{\mathbb{D}}}{\mathrm{D}}$ pouring the plates they are supported on level blocks 3 placed under the glass as the metal flanges may noto be level.

A plate containing a deeper layer of medium requires a longer incubation time to give clear-cut ${ }^{-}$ zones, but this leads to background haziness ando larger zone diameters which may merge with each other and may also influence each other. Theo figures shown in Table III illustrate the effect of

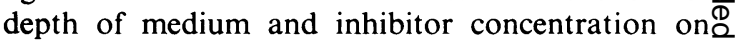
zone diameter.

\section{Spacing of Dises}

The growth zone edge represents the point to which sufficient phenylalanine has diffused over a period? of time to enable growth to occur. The diffusion of $\frac{}{3}$ phenylalanine extends under certain conditions far beyond the growth zone edge and therefore allowance must be made in spacing for the summation

\begin{tabular}{|c|c|c|c|c|c|c|c|c|}
\hline \multirow{2}{*}{ 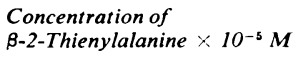 } & \multicolumn{7}{|c|}{ Depth of Medium ( $\mathrm{mm})$} & \multirow{2}{*}{$\begin{array}{l}\text { Phenylalanine Content of Blood } \\
(\mathrm{mg} / 100 \mathrm{ml})\end{array}$} \\
\hline & 3 & $4 \cdot 5$ & 6 & $7 \cdot 5$ & 9 & $10 \cdot 5$ & 12 & \\
\hline \multirow[t]{2}{*}{$8 \cdot 0$} & 10.0 & $8 \cdot 0$ & $\mathrm{NG}^{1}$ & NG & NG & NG & NG & 4 \\
\hline & 16.0 & 14.0 & $12 \cdot 0$ & 10.0 & $9 \cdot 0$ & $A^{2}$ & A & 10 \\
\hline \multirow[t]{2}{*}{$4 \cdot 0$} & $15 \cdot 5$ & $11 \cdot 5$ & $10 \cdot 5$ & $9 \cdot 5$ & $9 \cdot 0$ & $8 \cdot 0$ & $8 \cdot 0$ & 4 \\
\hline & $21 \cdot 0$ & $17 \cdot 5$ & $16 \cdot 0$ & $15 \cdot 0$ & 14.5 & $14 \cdot 0$ & $14 \cdot 0$ & 10 \\
\hline $2 \cdot 0$ & $19 \cdot 0$ & $17 \cdot 5$ & $15 \cdot 5$ & $14 \cdot 5$ & 140 & $13 \cdot 5$ & 13.5 & 4 \\
\hline \multirow[t]{2}{*}{$1 \cdot 0$} & 26.0 & $25 \cdot 5$ & 25.0 & $24 \cdot 5$ & $23 \cdot 5$ & $\mathrm{~B}^{3}$ & B & 4 \\
\hline & $32 \cdot 5$ & 30.5 & $28 \cdot 5$ & $27 \cdot 5$ & 27.0 & B & B & 10 \\
\hline
\end{tabular}

Table III Growth zone diameters $(\mathrm{mm})$ of $4 \mathrm{mg}$ and $10 \mathrm{mg}$ discs with changes in inhibitor concentration and in depth of medium

${ }^{1} \mathrm{NG}=$ no growth $;{ }^{2} \mathrm{~A}=$ very slight growth $;{ }^{3} \mathrm{~B}=$ indistinct zone edge, hazy background. 
effect of adjacent discs. This may well be sufficient for growth to occur between discs and hence a hazy background may result on a medium which appears satisfactory when testing solitary discs. This effect can be shown by carrying out tests with discs placed at varying distances apart. The growth zone, which is circular with well separated discs, becomes elliptic when the discs are placed sufficiently close for phenylalanine from one disc to affect the growth zone of the other.

One hundred discs can be accommodated in a $10 \times 10$ formation on a 10 in. $\times 10$ in. assay plate. Positioning of discs on the medium is assisted by the use of a template. This consists of a card with printed dots which are visible through the glass base of the plate. In routine screening it is not necessary to incorporate a wide range of standard discs. In this laboratory, 4, 6, and $8 \mathrm{mg}$ discs are included with each batch of specimens. The $4 \mathrm{mg}$ discs are placed at each corner of the plate to provide a check on the uniformity of the depth of the agar. The 6 and $8 \mathrm{mg}$ discs are sited both on the top line and on the bottom line between the $4 \mathrm{mg}$ standards, thus leaving the intermediate eight lines free for the uninterrupted placing of the test discs. However, if plastic trays are used it is essential to place extra standards, especially in the centre of the plate, to guard against variations of depth due to warping of the tray. Standard discs should be autoclaved with each batch of tests to allow for any possible reduction in the phenylalanine content by heating, and spare standard discs should be included with each autoclaved batch in case the second disc on any test card has to be tested at a subsequent date.

\section{Pre-incubation Diffusion}

Assay plates are usually incubated shortly after the blood discs have been added. Duplicate discs were added to assay plates, which were then left at room temperature for varying times before incubation to see if the pre-incubation diffusion of phenylalanine would result in a more even growth around the disc. No significant advantages were found; but it may be useful, when dealing with large numbers of specimens, to delay incubation of the plate after the discs have been added in order to bring the reading on the following day to a more convenient time.

\section{Concentration of Agar in Medium}

Increasing the concentration of agar in the medium produced only marginal reductions in the diameter of growth zones. There was no sharper definition of the zone edge.

\section{Effect of Antibiotics and Contaminating Substances}

Contamination with disinfectants may cause total suppression of growth and occasionally specimens are found to have suppression of growth from other causes. In many such cases investigated, the baby or mother had been receiving an antibacterial drug, usually ampiclox. From some discs no growth at all was obtained but more frequently an inner clear zone was found with a circle of growth outside it denoting diffusion of phenylalanine beyond the range of effect of the inhibitory substance.

It seemed probable that a high phenylalanine specimen would be recognized by diffusion beyond the clear zone, but experiments were carried out to ascertain the effects of the following antibiotics likely to be encountered in neonatal testing: (1) ampicillin, (2) cloxacillin, (3) ampiclox, a combination of (1) and (2), (4) tetracyclines, (5) benzyl penicillin, (6) cephaloridine, and (7) gentamicin.

Fisch, Anthony, Bauer, and Bruhl (1968) studied the effects of certain of these antibiotics on the results of the Guthrie test when given to phenylketonuric patients and when added to blood samples. Although the $B$. subtilis strain used was very sensitive to several antibiotics they found little difference in the results in the two experiments.

In our experiments phenylalanine was added to blood to give concentrations of $16,8,4$, and $2 \mathrm{mg} / 100 \mathrm{ml}$. To aliquots of these samples were added the antibiotics listed above to give drug concentrations higher than the maximum levels likely to be encountered. Samples were also prepared containing one tenth of these concentrations to simulate conditions more frequently found in clinical practice.

Guthrie tests were carried out on these samples after treatment with glacial acetic acid fumes or autoclaving. The results are shown in Table IV and Figure 2.

In all cases when any growth occurred in samples with antibiotics the growth zone diameters approximated to those obtained with the same samples without antibiotics. The figures given in the tables as 'zones of inhibition' are the diameters of inner clear zones due to the effect of the antibiotic. Discs with growth zones only slightly larger than the inner inhibited zones showed some reduction in the density of growth but the extent of the growth could still be measured and the diameters were unaffected.

On repeating the experiment after storing the dried blood discs for eight days at room temperature, the antibiotic effect was found to be reduced and with some discs, which previously had shown inner clear zones, normal growth was obtained. These findings are in keeping with experience that many 


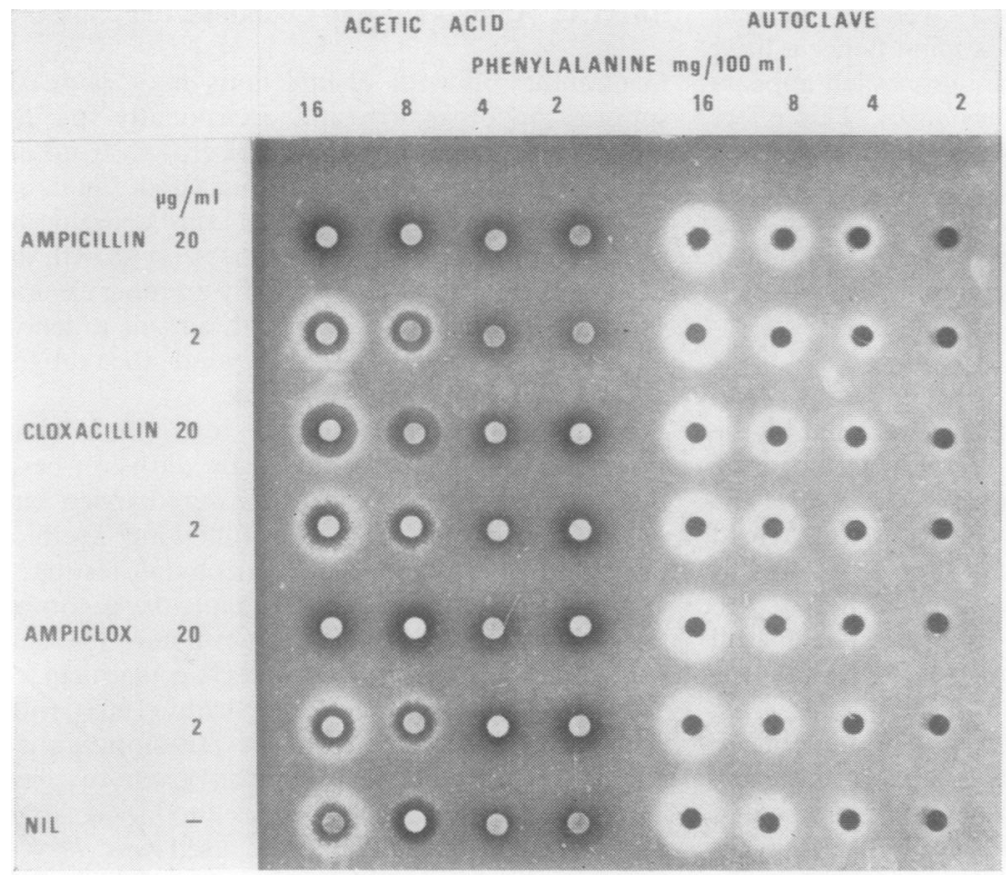

Fig. 2 Inhibition assay of blood samples containing added $\stackrel{\mathbb{Q}}{\stackrel{\mathbb{Q}}{2}}$ phenylalanine and antibiotics after autoclaving or acetic acid treatment

A

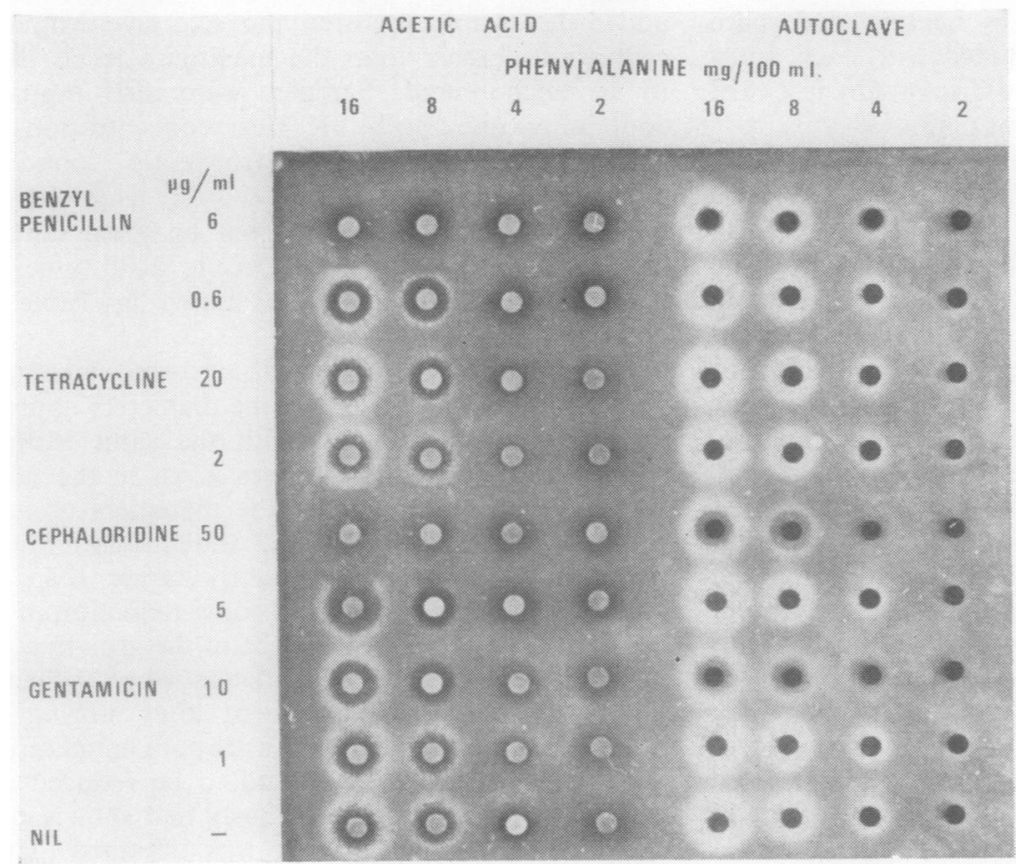




\begin{tabular}{|c|c|c|c|c|c|c|c|c|c|}
\hline \multirow[t]{3}{*}{ Antibiotic $(\mu g / m l)$} & & \multicolumn{8}{|c|}{ Phenylalanine $(\mathrm{mg} / 100 \mathrm{ml})$} \\
\hline & & \multicolumn{4}{|c|}{ Acetic Acid } & \multicolumn{4}{|c|}{ Autoclave } \\
\hline & & 16 & 8 & 4 & 2 & 16 & 8 & 4 & 2 \\
\hline \multicolumn{10}{|c|}{ Zones of inhibition ( $\mathrm{mm}$ ) } \\
\hline Benzyl penicillin & $\begin{array}{l}6 \\
0.6\end{array}$ & $\begin{array}{l}N^{1} \\
13\end{array}$ & $\begin{array}{l}\text { NG } \\
13\end{array}$ & $\begin{array}{l}\text { NG } \\
\text { NG }\end{array}$ & $\begin{array}{l}\text { NG } \\
\text { NG }\end{array}$ & $\sqrt{V}^{8 \cdot 5}$ & $\sqrt{V}^{8 \cdot 5}$ & $\sqrt{V}^{8 \cdot 5}$ & $\underset{\sqrt{ }}{\text { NG }}$ \\
\hline \multirow{2}{*}{ Tetracycline } & 20 & $\sqrt{ }^{2}$ & $\sqrt{ }$ & $\sqrt{ }$ & $\sqrt{ }$ & $\sqrt{ }$ & $\sqrt{ }$ & $\sqrt{ }$ & $\sqrt{ }$ \\
\hline & $\begin{array}{r}2 \\
50\end{array}$ & $\begin{array}{l}\sqrt{ } \\
\text { NG }\end{array}$ & $\begin{array}{l}\sqrt{ } \\
\text { NG }\end{array}$ & $\begin{array}{l}\sqrt{ } \\
\text { NG }\end{array}$ & $\sqrt{N G}$ & $\sqrt{ }$ & $\sqrt{ }$ & $\sqrt{N G}$ & $\sqrt{N G}$ \\
\hline Cephaloridine & 5 & 15 & $12 \cdot 5$ & NG & NG & $\sqrt{\sqrt{11} \cdot 5}$ & $\sqrt[12 \cdot 5]{\sqrt{ }}$ & $\underset{\sqrt{ }}{\mathbf{N G}}$ & $\underset{\sqrt{ }}{N G}$ \\
\hline \multirow[t]{2}{*}{ Gentamicin } & 10 & $12 \cdot 5$ & 12 & 13 & NG & 11 & 11 & $10 \cdot 5$ & NG \\
\hline & $\begin{array}{r}1 \\
20\end{array}$ & NG & $\stackrel{\sqrt{ }}{\text { NG }}$ & $\mathbf{N}^{\prime}$ & $\begin{array}{l}\sqrt{N G} \\
\text { NG }\end{array}$ & $\sqrt{7 \cdot 5}$ & $\sqrt{7 \cdot 5}$ & $\sqrt{7 \cdot 5}$ & $\sqrt{7 \cdot 5}$ \\
\hline Ampicillin & 2 & $12 \cdot 5$ & $11 \cdot 5$ & NG & $\sqrt{ }$ & $\sqrt{ }$ & $\sqrt{ }$ & $\sqrt{ }$ & $\sqrt{ }$ \\
\hline \multirow[t]{2}{*}{ Cloxacillin } & 20 & $16 \cdot 5$ & $15 \cdot 5$ & NG & NG & $\sqrt{ }$ & $\sqrt{ }$ & $v^{\prime}$ & $\sqrt{ }$ \\
\hline & 2 & $\sqrt{ }$ & $\sqrt{ }$ & $\sqrt{ }$ & $\sqrt{ }$ & $\sqrt{ }$ & $\sqrt{ }$ & $\sqrt{ }$ & $\sqrt{ }$ \\
\hline \multirow[t]{2}{*}{ Ampiclox } & 20 & NG & NG & NG & NG & $\sqrt{ }$ & $\sqrt{ }$ & $\sqrt{ }$ & $\sqrt{ }$ \\
\hline \multirow{2}{*}{\multicolumn{2}{|c|}{$\begin{array}{l}\text { Growth zone diameters }(\mathrm{mm}) \\
\text { No antibacterial drug }\end{array}$}} & 11 & 10.5 & $11 \cdot 5$ & NG & $\sqrt{ }$ & $\sqrt{ }$ & $\sqrt{ }$ & $\sqrt{ }$ \\
\hline & & 24 & $20 \cdot 5$ & 17 & $11 \cdot 5$ & 23 & 19 & 14.5 & 11 \\
\hline
\end{tabular}

Table IV Diameters of growth zones and zones of inhibition obtained from blood samples containing added phenylalanine and antibiotics after autoclaving or acetic acid treatment

${ }^{1} \mathrm{NG}=$ no growth; ${ }^{2} \sqrt{ }=$ normal growth with no inhibitory zore.

antibiotic sensitivity discs deteriorate rapidly on storage at room temperature.

The above results indicated that the effect of high concentrations of certain antibiotics could be detected in Guthrie test screening but the presence of these antibiotics, at levels unlikely to be exceeded clinically, did not result in total suppression of growth at phenylalanine levels above $4 \mathrm{mg} / 100 \mathrm{ml}$ if the sample had been autoclaved. Provided the test conditions are arranged to give some detectable growth at low phenylalanine levels, false negative results should not occur from this cause.

It is clear that treatment with acetic acid is less effective than autoclaving in reducing the effect of antibacterial substances. A few discs have been seen with very small inhibitory zones where no history of antibacterial therapy in the baby or the mother was obtainable. It seems possible that naturally occurring substances active against $B$. subtilis may be present in some bloods.

\section{Standards}

A direct comparison between commercially available standards was not possible because of the variety of papers used. It is possible only to compare standards on the same grade of paper and the specification of the paper was not usually stated by the manufacturers. An indication of the confusion which could arise from the use of standards prepared on dissimilar papers is given in Table $V$ which shows results of tests on standards from three different sources.

\begin{tabular}{|c|c|c|c|}
\hline \multirow{2}{*}{$\begin{array}{l}\text { Phenylalanine } \\
\text { Content } \\
(\mathrm{mg} / 100 \mathrm{ml})\end{array}$} & \multicolumn{3}{|l|}{ Standard } \\
\hline & Source $A$ & Source B & Source C \\
\hline $\begin{array}{r}10 \\
8 \\
6 \\
4 \\
2 \\
1\end{array}$ & $\begin{array}{l}21 \cdot 5 \\
20 \cdot 5 \\
18 \\
16 \\
13 \cdot 5 \\
12 \cdot 5\end{array}$ & $\begin{array}{l}23 \\
23 \\
21 \cdot 5 \\
18 \cdot 5 \\
16 \cdot 5 \\
15\end{array}$ & $\begin{array}{l}\overline{23} \cdot 5 \\
23 \\
22 \\
17 \cdot 5 \\
\text { Faint growth }\end{array}$ \\
\hline
\end{tabular}

Table V Comparison of standards from different sources ${ }^{1}$

${ }^{1}$ Growth zone diameters in $\mathrm{mm}$

Variation also occurred in the presentation of standards. Some standards were supplied as strips of paper entirely soaked with blood; others were available as punched discs which rendered treatment by autoclaving, in a similar manner to the test samples, impossible. In addition some standards were presented as discs impregnated with aqueous solutions. In view of the lack of uniformity and the risk of effects not within our control, it was decided to prepare our own standard blood spotted onto Whatman ET 31.

Standards may be prepared by estimating phenylalanine fluorimetrically in outdated blood from the blood bank and adding phenylalanine to give the required concentration. Stern (personal communication) has pointed out some theoretical objections to this: (1) Difficulties arise in the correlation of results obtained in fluorimetric analysis of stored blood with those obtained by inhibition assay 
because some amino acids and phenylalanine-containing peptides formed by degradation of protein interfere with the fluorimetric assay while some phenylalanine derivatives are estimated by the Guthrie test. (2) It is not entirely satisfactory to compare fluorimetric results obtained using plasma and Guthrie results using whole blood because the partition of added phenylalanine between cells and plasma in blood may not be similar. Woolf gives a cell:plasma ratio of $1 \cdot 1: 1$ (personal communication). In addition, if estimations are carried out by any process involving precipitation of protein, due allowance must be made for the associated volume changes.

Aqueous standards were considered but rejected because the resultant growth appeared dissimilar to the denser growth of the blood tests and comparison of tests using such samples showed that similar growth zones were obtained only when the phenylalanine concentration in the aqueous solution was much higher than in the blood specimen.

Experiments were carried out to determine a suitable method of reducing the amount of phenylalanine and other substances in expired transfusion blood, which in some instances contained over 3 $\mathrm{mg} / 100 \mathrm{ml}$. Aliquots of the blood were treated in one of the following ways: (1) blood dialysed in Visking tubing against saline at $4^{\circ} \mathrm{C}$; (2) cells washed in twice their volume of saline three times; (3) cells washed twice in saline, resuspended in saline at $4{ }^{\circ} \mathrm{C}$ overnight and washed again in saline. Freeze-dried pooled plasma was reconstituted with distilled water and dialysed in Visking tubing against saline at $4^{\circ} \mathrm{C}$. This was used to adjust the packed cell volume of each of the treated blood samples to that of the untreated stored blood. Guthrie tests and fluorimetric estimations were carried out on all samples.

The results indicated that some reduction occurred in the phenylalanine content of the dialysed whole blood and a considerable reduction occurred in that of the washed cells, especially those which had stood overnight in saline. The reconstituted and dialysed plasma gave no growth on Guthrie testing and $0.1 \mathrm{mg} / 100 \mathrm{ml}$ fluorimetrically; the final reconstituted blood gave no growth in the Guthrie test and values of about $0.3 \mathrm{mg} / 100 \mathrm{ml}$ in fluorimetric analysis.

Standards are now prepared by washing cells as above in saline, reconstituting with dialysed plasma to a PCV of $57 \%$ (approximate to that of a baby 1 week old), and the addition of phenylalanine.

\section{Preparation of Medium}

1 Oxoid PKU agar, without inhibitor, dehydrated powder

$2 \beta$-2-Thienylalanine $0.01 \mathrm{M}$ stored at $4^{\circ} \mathrm{C}$ (stock solution)
$3 \beta$-2-Thienylalanine $0.001 \mathrm{M}$ prepared weekly, stored at $4^{\circ} \mathrm{C}$ (working solution)

4 Sterile distilled water

5 Spore suspension prepared as detailed elsewhere.

For one $10 \mathrm{in} . \times 10 \mathrm{in}$. assay plate, $8.0 \mathrm{ml}$ of the dilute inhibitor solution is added to $190 \mathrm{ml}$ of sterile distilled water at $60^{\circ} \mathrm{C}$ in a conical flask. Then $9 \mathrm{~g}$ of the agar is added and the mixture allowed to stand for 15 minutes. It is slowly brought to the boil on a hotplate with gentle heat. After approximately two minutes' boiling, the medium becomes clear and boiling is continued for a further two minutes. When the medium has cooled to $60^{\circ} \mathrm{C}, 3.0 \mathrm{ml}$ of spore suspension is added and the contents of the flask are well mixed. Care is taken to avoid bubbles and the medium is poured into an assay plate and allowed to solidify.

\section{Interpretation of Results}

The levels used in this laboratory are: (1) Under $4 \mathrm{mg} / 100 \mathrm{ml}$ the test may be reported 'negative', provided some growth has occurred. (2) From 4 to $6 \mathrm{mg} / 100 \mathrm{ml}$ is found in about $0.5 \%$ of $\overrightarrow{0}$ tests when carried out on the sixth day of life and is usually associated with premature babies. A request is made for a repeat specimen to be taken on the fourteenth day, when it is nearly always found to be negative. To avoid parental anxiety, particular care is taken when first reporting such results to emphasize the probable negative outcome of the repeat test and results of these tests are reported by telephone. (3) Above $6 \mathrm{mg} / 100 \mathrm{ml}$ individual action is taken according to the actual figure obtained and the clinical circumstances. This involves notification of the result to the collection centre, the general practitioner, and the district medical officer of health so that further investigations are initiated.

\section{Discussion}

Because of the low incidence of phenylketonuria, inaccuracies of technique in the Guthrie test might not become apparent for a considerable time unless the methodology of the test were examined critically. O Very high levels of phenylalanine would probably $N$ be recognized in spite of poor techniques, but there $N$ are recorded cases (Hsia, 1967) of phenylketonuria with initial phenylalanine levels only slightly raised which could be missed. There is growing experience also of babies with disturbances of amino acid $\cong$ metabolism, often transient, but of considerable interest, and sometimes requiring treatment, which are detected initially by slightly raised Guthrie test results.

Some misgivings have been expressed regarding , 
the testing of babies receiving antibiotics and it has been suggested that the collection of the Guthrie test sample from these babies should be delayed. In view of the large numbers of babies who receive antibiotics such a practice would lead to administrative difficulties and many of these babies would escape subsequent testing. Furthermore, the experiments detailed above indicate that, provided the samples have been autoclaved, raised levels of phenylalanine should not be masked by the usual clinical levels of the listed antibiotics. Very high concentration of antibacterial drugs can result in complete suppression of growth but in these cases the tests would not be declared negative if the "no result without growth' rule had been followed.

Plastic trays are widely used as assay plates in Guthrie testing and may be very suitable but some trays designed for other purposes tend to warp with repeated use. The variation in depth of medium which results from a faulty tray may be guarded against by frequent inspection of the dish and the placing of extra standard discs on the medium; but the use of metal and glass assay plates is more satisfactory. The extra capital cost is small. The assay plates last longer and they may be sterilized, if necessary, by autoclaving.

Contamination has not been reported generally to be a problem in Guthrie testing but in this laboratory the assay plates are routinely autoclaved daily with batches of other laboratory material.

Costing of a service of this kind is notoriously difficult, but an attempt has been made to price materials, staff, and communications as far as possible. It is impossible to evaluate the time of field workers collecting samples but the laboratory side of the service can be costed with reasonable accuracy.

Considerable saving can be made by the use of laboratory-produced materials, eg, $B$. subtilis spore suspension and collecting cards, and the tendency to organize neonatal screening services on a regional basis will reduce, it is hoped, the use of relatively expensive commercial kits. As a guide, the following figures represent the cost per test for the Region for materials. Collection cards with polythene envelopes $0.17 \mathrm{p}$; medium, $0.16 \mathrm{p}$; request forms and printed envelopes, $0.08 \mathrm{p}$, giving a figure for laboratory materials alone of $0.41 \mathrm{p}$. However, when to this is added the cost of postage, telephone, staff salaries, and lancets, the total cost rises to $11 \mathrm{p}$.

A fuller analysis of costs is given in a separate publication describing the administrative aspects of the regional scheme.

The collection card now available from the Department of Health has not been adopted for this region, as a satisfactory card employing Whatman ET 31 was already being used. The large size of the new card precluded its use in the existing laboratory arrangements and, furthermore, the layout of the card was not suitable for our reporting procedure, which was organized at the request of the medical officers of health of the region.

The Department of Health circular HM (69) 72 states that the Guthrie test should be carried out as early as possible between the infant's sixth and fourteenth day of life. This long period appeared to us to allow too much latitude in the day of testing and to increase the chances of babies escaping the net altogether.

The decision to collect samples throughout this region on the sixth day of life was strengthened by the following considerations: first, infants are not usually discharged from hospital until at least the seventh day, except for the 48-hour discharge babies who are firmly in the care of the domiciliary services by the sixth day; secondly, most babies pass from the care of the midwife to the health visitor at about the 10th day. It therefore appeared that the sixth day would be within a period when the infant was least likely to be changing from one authority's care to another, thus ensuring more certainly that the test had been done. The instruction that the tests should be carried out on a particular day of life has advantages in a scheme involving many operators not very closely connected in some cases with each other.

The practice of returning blood samples, with phenylalanine levels between 20 and $40 \mathrm{mg} / 100 \mathrm{ml}$ under fictitious names from collection centres to check the screening procedure (Scotland, South-East Hospital Region, Consultant Paediatricians and Medical Officers, 1968), was not adopted by this laboratory. It is felt that this does little more than check the postal system, as special arrangements have to be made at the sending centre for these raised samples and the routine arrangements are not tested; at the laboratory it is difficult to imagine that a level over $20 \mathrm{mg} / 100 \mathrm{ml}$ could escape detection. In this laboratory the reserve $4 \mathrm{mg}$ disc included with each batch of specimens is used occasionally when retesting a sample as detailed elsewhere. This $4 \mathrm{mg}$ sample, appearing in occasional batches in random positions on the assay plate, is a useful check of technical vigilance.

The Department of Health circular HM (69) 72 states that levels of phenylalanine below $6 \mathrm{mg} / 100 \mathrm{ml}$ when tested between the infants' sixth and fourteenth days of life may be regarded as negative. This view is not universally held and the re-testing of babies with blood phenylalanine levels above $4 \mathrm{mg} / 100 \mathrm{ml}$ is recommended. Although the outcome of re-tests from babies with slightly raised results will in all probability be negative for phenylketonuria, the 
extra repeat tests necessitated by adopting the lower level are very few and must be weighed against the value of the information obtained.

The selection of $4 \mathrm{mg} / 100 \mathrm{ml}$ as the dividing point resulted in a retake rate of $0.67 \%$. With a $6 \mathrm{mg} / 100 \mathrm{ml}$ figure being used, the re-test figure was $0.22 \%$. Therefore using the lower limit the additional retests required per year $(50,000$ babies) amounted to 225. The re-testing and investigation of these babies is now beginning to yield useful information on normal and abnormal biochemical states in the neonatal period.

Efforts are being made to automate certain stages of the test, notably the punching and placing of discs on the plates. So far no satisfactory machine has been seen which fulfils all the requirements for the identification of specimens and elimination of possible errors at a reasonable price. It is hoped that in time such a machine will be produced.

When using the Guthrie test, the factors detailed above may insidiously affect the result. Knowledge of the variables within the Guthrie test leads to greater technical accuracy, permits the technique to be adjusted to suit the purpose for which the test is being used, and enables sources of error to be recognized.

It is, therefore, recommended that screening procedures of this nature are only undertaken in laboratories able to deal with large numbers of samples and equipped to offer biochemical facilities for the detailed investigation of selected cases. This could yield, in addition, useful statistical information and 50,000 samples per year may well be an acceptable minimum number.

The role of the screening laboratory should not be only to test large numbers of samples. The planning and maintenance of a screening service depends on attention being paid to all aspects of the testing facilities, and from its central position the laboratory is well fitted to observe the scheme as a whole, indicate any situation requiring attention, and offer assistance where needed. The laboratory, therefore, in addition to testing specimens should be willing to act as adviser and coordinator of the efforts of the many people concerned and close cooperation with those in day-to-day contact with the babies and mothers has proved of great value in the smooth running of the service.

\section{Addendum}

Since this paper was written, $3 \mathrm{~mm}$ (instead of $t$ in.) blood discs are being increasingly used for inhibition assays. This permits more tests to be carried out on a single spot of blood. To achieve maximum sensitivity of the test, the amount of inhibitor used should be reduced when using the smaller discs. A final concentration of $1.5 \times 10^{-5} \mathrm{M}$ has been found satisfactory.

We are pleased to record the willing assistance given by many people during the setting up of the South West Metropolitan Regional Infant Screening Service.

The staff of maternity departments, both hospital and domiciliary, are key figures in the smooth running of the scheme; and ready assistance has been given also by medical officers of health and their staff. We are particularly indebted to the staff of the Health Department of the London Borough of Sutton where the service first began.

We are grateful to Dr E. Lund, Statens Seruminstitut, Copenhagen, for information on Guthrie testing in Denmark and for samples of $B$. subtilis strains.

We are indebted to many paper manufacturers for technical information and to the following suppliers of Guthrie testing materials who kindly gave samples for trials: Baird \& Tatlock Ltd (Bacto-Difco), Becton Dickinson UK Ltd (Baltimore Biological Laboratories), Mast Laboratories Ltd, Anderman \& Co, Ltd (Merckotest. E. Merck, A.G. of Darmstadt). We thank Mr E. Y. Bridson, Oxoid Ltd., who cooperated closely in the preparation of a suitable PKU medium.

We thank our colleagues Dr J. Stern and Dr R. J. Holt, for the sound advice given in the establishment of this service. In addition Dr Stern has provided fluorimetric analysis of many samples and Dr Holt has given valuable criticism of this paper.

\section{References}

Department of Health and Social Security Screening for early detection of phenylketonuria. Dept. of Health and Social Security Circular. HM (69) 72.

Efron, M. L., Young, D., Moser, H. W., and MacCready, R. A. (1964). A simple chromatographic screening test for the $\supsetneq$ detection of disorders of amino acid metabolism. New Engl. J. 응 Med., 270, 1378-1383.

Fisch, R. O., Anthony, B. F., Bauer, H., and Bruhl, H. H. (1968). D The effect of antibiotics on the results of the Guthrie test given to phenylketonuric patients. J. Pediat., 73, 685-689.

Guthrie, R., and Susi, A. (1963). A simple phenylalanine method for $N$ detecting phenylketonuria in large populations of newborn infants. Pediatrics, 32, 338-343.

Hill, J. B., Summer, G. K., Pender, M. W., and Roszel, N. O. (1965). A. automated procedure for blood phenylalanine. Clin. Chem., $\omega$ 11, 541-546.

Hsia, D. Y.-Y. (1967). Phenylketonuria. Develop. Med. Child. Neurol., 9, 531-540.

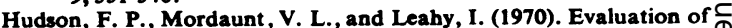
treatment begun in first three months of life in 184 cases of $\mathrm{C}$ phenylketonuria. Arch. Dis. Child, 45, 5-12.

Kennedy, R. (1969). A simple device for the rapid plating of auto- $\square$ claved blood spots for the Guthrie test. J. med. Lab. Technol., 26, 269-270.

Medical Research Council, Working Party on Phenylketonuria (1968). Present status of different mass screening procedures for (D) phenylketonuria. Brit. med. J., 4, 7-13. 
Scheel, C., and Berry, H. K. (1962). Comparison of serum phenylalanine levels with growth in Guthrie's inhibition assay in newborn infants. J. Pediat., 61, 610-616.

Scotland, Consultant Paediatricians and Medical Officers of Health (1968). Population screening by Guthrie test for phenylketonuria in South-East Scotland. Brit. med. J., 1, 674-676.

Searle, B., Mijuskovic, M. B., Widelock, D., and Davidow, B. (1967). A manual fluorometric paper disc method for detecting phenylketonuria. Clin. Chem., 13, 621-625.

Smith and Gordon, unpublished data. Cited in Bergey (1951). Manual of Determinative Bacteriology, 7th ed., p. 620. Williams and Wilkins, Baltimore.

Wainer, S. C., and Metzger, L. F. (1967). A simple device for rapid plating of phenylketonuria specimens for the Guthrie bacterial inhibition assay. Amer. J. clin. Path., 48, 255-256.

Suppliers of Apparatue and Materials

MEDIUM

PKU test agar, code CM 365: Oxoid Ltd, Southwark Bridge Road,
L.ondon SE1

B-2-THIENYLALANINE

Sigma Chemical Co Ltd, 12 Lettice Street, London SW6

ASSAY PLATES

Mast Laboratories Ltd, 38 Queensland Street, Liverpool 7

CHROMATOGRAPHY PAPER

Whatman ET 31: W. \& R. Balston Ltd,Sprinefield Mill, Maidstone, Kent. Laboratory stockists: C. E. Payne \& Sons Ltd, 131 Clapham High Street, London SW4

DUPLCATING INK

HMSO Ink, Duplicating, grey-black, stock item Code no, 42-47

PRINIING INT

Adana 731 Black No. 2

PRINIING EQUTPMENT

Adana (Printing Machines) Ltd, 15-19 Chusch Street, Twickenham, Middlesex

DISPOSABLE BLOOD LANCETS

Armour Pharmaceutical Co, Ltd, Hampden Park, Eastbourne, Sussex Steriseal Ltd, Redditch, Worcestershire

HAND PUNCH

Maun Induatriea Ltd, Moor Lane, Mansfield, Notts 